\title{
In vivo study of Fagonia Cretica Linn toxicity in Sudan
}

\author{
Omer Abbas Mohamad Jahala ${ }^{1 *}$, Omer Musa Izzeldin ${ }^{2}$, Rashid Eltayeb Abdalla ${ }^{3}$ \\ ${ }^{1}$ Faculty of Medical Laboratory Science, Shendi University, Sudan \\ ${ }^{2}$ Faculties of Science and Technology. Omdurman Islamic University, Sudan \\ ${ }^{3}$ Faculty of Medicine and Surgery, Shendi University and School of pharmacy, Ahfad University for Women, Sudan \\ *Corresponding author E-mail: Mehina2007@yahoo.com
}

\begin{abstract}
Purpose: The study targeted to determine the toxicological effects of ethanolic extracts of Fagonia cretica linn plant in Khartoum, Omdurman, and Shendi towns in Sudan during (January - February) 2013.

Objectives: To determine the LD50 of the plant and to study the effects of the plant on the body weight, on vital organ weights, and anesthesia induction time effects.

Methods: Different methods were adopted to achieve the objectives of this study, including Harborne methods for extraction. A total of (30), young adult Wistar rats, weighing $(42.6$ - 72.2) grams, fed standard rat food and water d libitum, were maintained at standard laboratory conditions. All rats were grouped into (3) groups, survived through the experiment by using the recommended progression doses of Fagonia cretica as $(175,550,1750,5000,5000, \& 5000) \mathrm{mg} / \mathrm{kg} /$ body weight screen shot of AOT425 statistical program of main test. Toxicological studies in vivo (LD50 determination) were done in all (3) groups of animals survive till the experiment was over after the administration of a selected high dose (5000) $\mathrm{mg} / \mathrm{kg} /$ body weight. Ethical approval had been obtained from Omdurman Islamic University, Faculty of Pharmacy, and Department of Pharmacology under the number-(01/0/2013).

Results: The LD50 is greater than $5000 \mathrm{mg} / \mathrm{kg}$, indicates the high safety of the plant. The ethanolic extract of Fagonia cretica in doses of $(100 \& 300 \mathrm{mg} / \mathrm{kg} /$ body weight) has no effects on the rats relative vital organ weight (heart, liver, kidneys, lung and spleen) during the study period of (14) days compared to the control group whilst the dose of (600) $\mathrm{mg} / \mathrm{kg} / \mathrm{body}$ weight change the liver somewhat from the relevant control, although, has no effects on the rats body weight during the study period of (14) days compared to the control group monitored at day $(0,7,14)$. The anesthesia induction time on rats, shows that the ethanolic extracts of the plant has neurological effect on rats.

Conclusion: The toxicological determination of LD50 was found to be greater than (5000) $\mathrm{mg} / \mathrm{Kg}$, estimated by the special statistical software with three stopping criteria based on long term outcomes. This is indicating wide safety margin of Fagonia ethanolic extract which encourages its use by humans for the treatment of many diseases..
\end{abstract}

Keywords: Fagonia Cretica Linn, Umm Showaika, Ethanolic Extraction, Toxicity.

\section{Introduction}

Life and diseases go together: Where there is life, diseases are bound to exist. Dependency and sustainability of man and animal life has been revolving around plants through uses as foods, fibers and shelter, but also plants have been used to control and ease diseases, therefore the use of the plants as medicines is an ancient and reliable practice (Sarfraz Khan Marwat et al 2010)The plant is a small spiny under shrub, mostly found in dry calcareous rocks throughout Pakistan. (Chopra, R.M.et al 1982, p.507) It is reputed to be a medicinal plant in scientific and folkloric literature and its medicinal values are well documented (Saied MA ET al.1969.p.41-43)

Vern names: (Ar) Umm Showaika, Sholib, UmmShok. Family : Zygophyllaceae. Habitat: Sandy hills (Quos), low land plains. In Sudan: ElMazroub, also widespread throughout Northern and central Sudan. (Gamal, E.B.ELGhazaliit et al 1994 .p.52-53) it is present abundantly in Shendi Region.

Universally: It is found in India, Pakistan, China, Bangladesh and Egypt.
Medicinal properties of the plant are attributed to its variety of active phytochemical constituents. In the last fifteen years, this plant and related species have been investigated mainly for the presence of flavonol and terpenoid glycosides. Most of the flavonol glycosides have been isolated from various Egyptian Fagonia species and their phylogenetic affinities have also been investigated. (Crack PJ.et al 2003.p.19-22) Several saponin glycosides have been separated and characterized. (Taylor CP. Et al 1993.p 555-563) Other constituents, such as docosyl docosanoate from hexane extract and water soluble proteins from aqueous extract of air-dried F. cretica plants have been isolated. Furthermore nahagenin (hederagnin, ursolic acid and pinitol from other Fagonia species have also been separated and characterized. (Titz F.et al.1969.p502-522). Antimicrobial activity of its flavonoid compounds has been explored previously. While the nutritive values of it and of other species growing wild in the Rajasthan region of India, have also been evaluated. (Paglia DE et al.1967.p.158-169). 


\section{Materials and methods}

This study was conducted in Omdurman Islamic University laboratories, Khartoum state, Sudan, during (January - February) 2013.

\subsection{Ethical-consideration}

An ethical approval had been obtained from Omdurman Islamic University, Faculty of Pharmacy, Department of Pharmacology, under the number $(01 / 0 / 2013)$.

The in vivo toxicological procedures involve the experimental animals and their care was conducted in compliance with the Guidelines for Care and Use of Laboratory Animals in Biomedical Research as promulgated by the Canadian Council of Animal Care (1984) and United States National Institutes of Health (1985).

The rats were fed a standard rat food (a mixture of flour, oil, meat and some vegetables) and water ad libitum and were maintained at standard laboratory conditions $(12 / 12 \mathrm{hr}$ dark/light cycle, $23 \pm 1$ ${ }^{\circ} \mathrm{C}$ temperature, and $55 \pm 3 \%$ humidity)

The Faonia cretica plants were collected from uncultivated and waste areas of Shendi town from near the Faculty of Medicine and Surgery, University of Shendi, Shendi town, River Nile state, Sudan in (January/February) 2011. These were authenticated by the Herbarium staff, Department of Botany, the Sudanese national centre for research, Khartoum, Sudan.

\subsection{Experiment-setup}

A voucher specimen was deposited in the Herbarium Department of Botany, the Sudanese national centre for research, Khartoum, Sudan for further reference. Extraction was carried out according to the method described by (Harborne J B, 1984.p.4-7).$^{9} 2000 \mathrm{~g}$ of plant sample was extracted successively with chloroform and 80 $\%$ ethanol using shaker apparatus; this was for about (72) hours for chloroform, and (5) days for ethanol. At last extracts were allowed to air till they were dried. A total of thirty, young adult Wistar rats, weighting (42.6- 72.7) grams were obtained from the Sudanese National Centre for Research, Khartoum, Sudan.. Before the oral doses administration experiment was beginning, rats were fasted overnight for $(14-16)$ hours. Group C, which was the control group, received (10) $\mathrm{ml} / \mathrm{kg}$ distilled water, orally, throughout the study period while Groups $(1,2,3)$ were orally administered single, daily doses, $(100,300$ and 600$) \mathrm{mg} / \mathrm{kg}$ of body weight, respectively of the Fagonia cretica ethanolic extract dissolved in distilled water $(1 \mathrm{gm} / 10 \mathrm{ml})$ for (14) days using acute oral toxicity (425) protocol. Acute oral toxicity study was conducted using the limit dose test of Up-and-Down Procedure according to Organization for Economic Cooperation and Development (OECD) at a limit dose of (5000) $\mathrm{mg} / \mathrm{kg}$ body weight per oral route. Behavioral manifestations of acute oral toxicity such as lethargy, anxiety, sleep, hyperactivity, changes in eyes, diarrheal etc, were also noted. All observations were systematically recorded and individual records being maintained for each rat. Single, daily doses, (100, 300 and 600$) \mathrm{mg} / \mathrm{kg}$ of body weight, respectively of the Fagonia cretica ethanolic extract dissolved in distilled water $(1 \mathrm{gm} / 10 \mathrm{ml})$ for (14) days using acute oral toxicity (425) protocol. Body weights of all rats were monitored using a sensitive balance at days ( $7 \& 14$ ) of the experiment; the obtained weights were statistically analyzed for comparison between test and control. The rat's weights of the (heart, liver, kidneys, lung and spleen) were monitored on the day (15) of the test, after scarifying the rats and the obtained weights were statistically analyzed, and compared with the control. The effects of ethanolic extract of Fagonia cretica in doses of $(100,300 \& 600 \mathrm{mg} / \mathrm{kg} /$ body weight) has been tested on the anesthesia induction time on rats after the study period of (14) days, compared to the control group.

Toxicological studies in vivo (LD50 determination) were done in all (3) groups of animals survive till the experiment was over after the administration of a selected high dose (5000) $\mathrm{mg} / \mathrm{kg} / \mathrm{body}$ weight (Table 1) although some signs were observed during the limit test such as lethargy, sleeping, hyperactivity, anxiety and sometimes inactivity.

\section{Results}

\subsection{General effects of fagonia cretica}

Statistical Program with its default setting.

Table 1: Sequence and Results of Limit Dose Test of Fagonia Cretica in Rats Using AOT425statpgm (Version: 1.0) (Acute Oral Toxicity (OECD Test Guideline 425)

\begin{tabular}{|c|c|c|c|c|c|}
\hline $\begin{array}{l}\text { Test } \\
\text { term } \\
\text { Seq. }\end{array}$ & Long-term & $\begin{array}{l}\text { Animal } \\
\text { ID } \\
\text { Result }\end{array}$ & Dose & $(\mathrm{mg} / \mathrm{kg})$ & $\begin{array}{l}\text { Short- } \\
\text { Result }\end{array}$ \\
\hline 1 & $\mathrm{O}$ & 1 & $\mathrm{O}$ & 5000 & \\
\hline 2 & $\mathrm{O}$ & 2 & $\mathrm{O}$ & 5000 & \\
\hline 3 & $\mathrm{O}$ & 3 & & 5000 & \\
\hline
\end{tabular}

$(\mathrm{X}=$ Death, $\mathrm{O}=$ Survival $)$

The LD50 is greater than $5000 \mathrm{mg} / \mathrm{kg}$.

Main test: All rats were survival through the experiment by using the recommended progression doses of Fagonia cretica as $(175$, $550,1750,5000,5000, \& 5000) \mathrm{mg} / \mathrm{kg} /$ body weight screen shot of AOT425 statistical program of main test.

i) Body weight effects:

The ethanolic extract of Fagonia cretica in doses of $(100,300 \&$ $600 \mathrm{mg} / \mathrm{kg} /$ body weight) has no effects on the rats body weight during the study period of (14) days compared to the control group monitored at day $(0,7 \& 14)$ this can be shown in Fig.( 1)

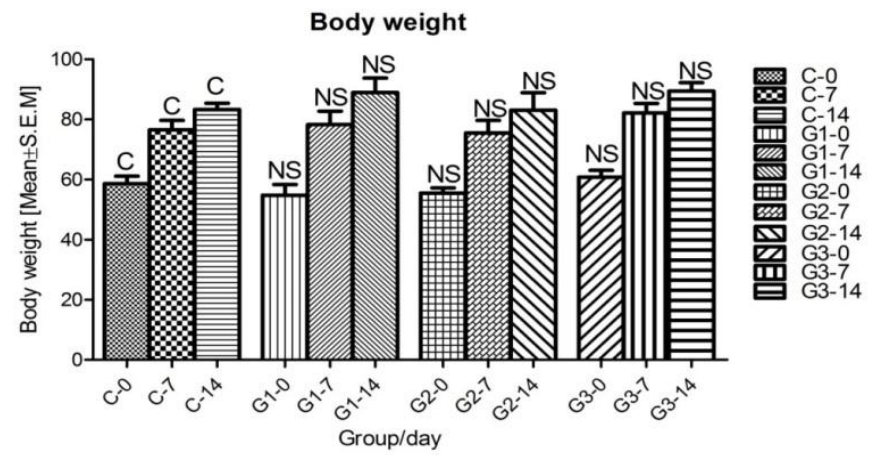

Fig. 1: Rats Body Weight during the Study Period Monitored at Day (0, 7 \& 14)

ii) Relative organ weight:

The ethanolic extract of Fagonia cretica in doses of $(100 \& 300$ $\mathrm{mg} / \mathrm{kg} /$ body weight) has no effects on the rats relative vital organ weight (heart, liver, kidneys, lung and spleen) during the study period of (14) days compared to the control group whilst the dose of $(600) \mathrm{mg} / \mathrm{kg} / \mathrm{body}$ weight change the liver somewhat from the relevant control.

Table 2: Effect of Ethanol Leaves Extract of Fagonia Cretica on the Relative Organs Weight and Lethality in Rats

\begin{tabular}{llllll}
\hline \multicolumn{5}{l}{ Relative organ weight $(\mathrm{g} / 100 \mathrm{~g}):$} \\
\hline $\begin{array}{l}\text { Doses } \\
(\mathrm{mg} / \mathrm{kg})\end{array}$ & Heart & Liver & Kidneys & Lung & Spleen \\
\hline Control & $0.35 \pm 0.01$ & $3.02 \pm 0.1$ & $0.66 \pm 0.03$ & $0.63 \pm 0.03$ & $0.20 \pm 0.02$ \\
100 & $0.35 \pm 0.02$ & $3.08 \pm 0.2$ & $0.68 \pm 0.03$ & $0.63 \pm 0.03$ & $0.20 \pm 0.01$ \\
300 & $0.33 \pm 0.02$ & $2.52 \pm 0.6$ & $0.63 \pm 0.03$ & $0.59 \pm 0.02$ & $0.19 \pm 0.01$ \\
600 & $0.36 \pm 0.02$ & $3.50 \pm 0.1 *$ & $0.66 \pm 0.02$ & $0.60 \pm 0.02$ & $0.20 \pm 0.01$ \\
\hline
\end{tabular}

iii) Anesthesia induction time effects:

The ethanolic extract of Fagonia cretica in doses of $(100 \& 300$ $\mathrm{mg} / \mathrm{kg} / \mathrm{body}$ weight) has no effects on the anesthesia induction time on rats after the study period of (14) days compared to the 
control group whilst the dose of (600) $\mathrm{mg} / \mathrm{kg} /$ body retard the anesthesia induction time on rats from the relevant control.

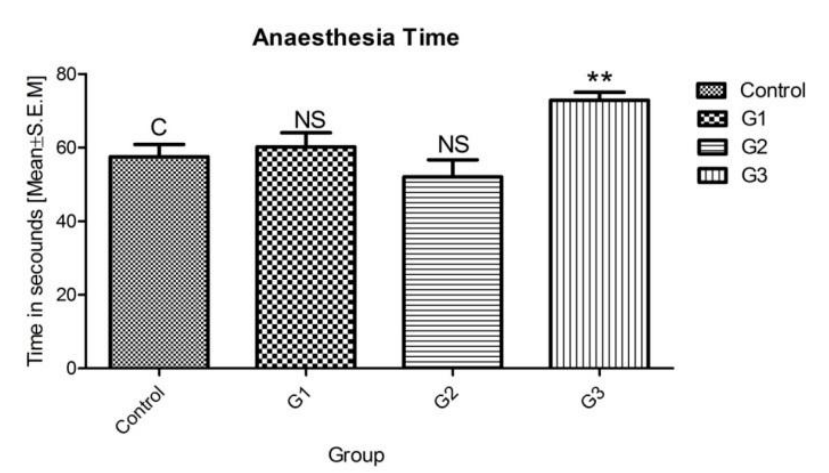

Fig.2: Anesthesia Induction Time for All Groups Using Diethyl Ether at the End of the Study (Day15)-Scarifying Date.

\section{Discussion}

This is an in vivo current study of the Sudanese Fagonia cretica linn that attempted to examine the toxicological effects, in order to fill the gap due to lack of such information's in Sudan. Nowadays, there is an increase and popular utilization of Fagonia for treating various health problems worldwide particularly in Pakistan, India, Far East and some other parts of the world including Sudan; hence there were limited scientific data available regarding its safety. This current case - control (14) days study evaluate the safety of Fagonia ethanolic extract using protocol 425 guidelines. Observations of rats' behaviors can be summarized as follows; rats were normally behaving during the adaptation period, after $(1 / 2)$ hours of dose administration some of them from different study groups develop inactivity, lethargy and asleep associated with some rats' hyperactivity. No behavioral changes occurred during the remaining test period. Statistical estimates based on long term outcomes. The LD50 is greater than $(5000) \mathrm{mg} / \mathrm{kg}$, which indicates the high safety of the plant, which encourages its use by human. This result is in line with (Matthew Lam 2012) who describes for the first time that an aqueous extract of Fagonia cretica shows potent in vitro cytotoxic activity towards breast cancer epithelial cell lines which was not seen towards normal mammary epithelial cells.

The ethanolic extract of Fagonia showed no effect in rat's body weight, which means absence of any substance that may cause increase in body weight.

No effects were observed on different vital organs using the tested doses, except for the large dose which has significantly increased the liver size, this hepatomegaly was not due to any pathological conditions because the macroscopically investigations showed normal liver texture, besides our liver function tests revealed normal functionality. This was in line with the traditional use in treating liver disorders (Avinash K, et al 2004.p.4-11). Also the literature survey revealed the presence of antioxidant effect that act as Hepato protective (Avanish, K et al 2004).

Only the dose of $(600) \mathrm{mg} / \mathrm{kg} / \mathrm{body}$ retarded the anesthesia induction time on rats, which means that the ethanolic extracts has a neurological effect, which agrees with (Avinash K et. Al., 2004).

\section{Conclusion}

The toxicological determination of LD50 was found to be greater than $(5000) \mathrm{mg} / \mathrm{Kg}$, estimated by the special statistical software with three stopping criteria based on long term outcomes. Which indicating wide safety margin for Fagonia ethanolic extract.

\section{Recommendations}

1) Further studies targeting the identification of the active phytochemical components of Fagonia cretica and their role of action are recommended.

2) Pharmaceutical formulation of Fagonia cretica as herbal medicine is highly recommended.

3) The LD50 of the plant showed a high margin of safety which encourages its use by human for the treatment of many diseases.

4) Further studies on the Sudanese Fagonia cretica as antioxidant, immune modulating agent, anticancerous, and antiinflammatory is also recommended.

\section{References}

[1] Paglia DE, Valentine WN: Studies on the quantitative and qualitative characterization of erythrocyte glutathione peroxidase. J Lab Clin. Med 1967, 70(1):158-169.

[2] Saied MA. Hamdard Pharmacopoeia of Easter Medicine. Hamdard. Academy, Karachi, Pakistan; 1969 phytochemical and biological activities of fagonia indica, p. 41-43.

[3] Titz F, Enzymatic method for quantitative determination of nanogram amounts of total and oxidized glutathione: Applications to mammalian blood and other tissues. Anal Biochem 1969, 27(3):502-522. http://dx.doi.org/10.1016/0003-2697 (69)90064-5.

[4] Chopra, R.M., Handa, K, L; Kapur, L, D., and Chopra, I.C., Indigenous Drugs of India. 2nd ed. India, Academic Press, New Delhi 1982, p. 507.

[5] J.B.Harborne. phytochemical methods a guide to modern techniques of plant analysis, Second Edition, Chapman and Hall, London, 1984, P. 4-7.

[6] Taylor CP, Weber ML: Effect of temperature on synaptic function after reduced oxygen and glucose in hippocampal slices. Neuroscience 1993, 52(3):555-562. http://dx.doi.org/10.1016/0306-4522 (93)904055.

[7] Gamal, E.B.ELGhazali, Mahgoub, S.ELToham. Awatif, A.B.ELEgami. Waiel. S.Abdalla, Medicinal Plants of the Sudan, 1994 National Centre for Research, Medicinal \& Aromatic Plants Research Institute, Khartoum, 52-53.

[8] Crack PJ, Taylor JM, de Haan JB, Kola I, Hertzog P, and Iannello RC: Glutathione peroxidase-1 contributes to the neuroprotection seen in the superoxide dismutase- 1 transgenic, journal of blood flow and metabolism. 2003, vol 23(1), page 19-22.

[9] Avinash K Rawal, Manohar G Muddeshwar and Saibal K Biswas, Rubia cordifolia, Fagonia cretica linn and Tinospora cordifolia exert neuroprotection by modulating the, antioxidant system in rat hippocampal slices subjected to oxygen glucose deprivation, BMC Complement Altern Me. 2004, 4:11. 10.1186/1472-6882-4-11. http://dx.doi.org/10.1186/1472-6882-4-11.

[10]Sarfraz Khan Marwat, Mir Ajab Khan, Mushtaq Ahmad, Muhammad Zafarand Fazal-ur Sarhad Ethnophytomedicines for treatment of various diseases in D. I. Khan district Int J Pharm Biomed Sci 2010.

[11] Matthew Lam Cytotoxic activity of Fagonia cretica against human breast cancer cells Doctor of Philosophy Aston University. April 2012. 